\title{
Smiles rearrangement for the synthesis of diarylamines
}

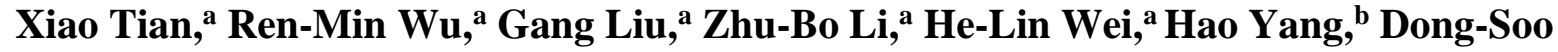 Shin, ${ }^{\mathrm{c}}$ Li-Ying Wang, and Hua Zuo ${ }^{\text {a* }}$}

${ }^{a}$ College of Pharmaceutical Sciences, Southwest University, Chongqing 400715, China

${ }^{b}$ College of Horticulture and Landscape Architecture, Southwest University, Chongqing 400715, China

${ }^{c}$ Department of Chemistry, Changwon National University, Changwon, 641-773, South Korea E-mail: zuohua@swu.edu.cn

\begin{abstract}
A protocol for the one-pot synthesis of diarylamines via Smiles rearrangement under microwave irradiation has been developed. Various diarylamines were effectively synthesized starting from readily available substituted phenols, arylamines and chloroacetyl chloride in moderate to good yields (58-92\%).
\end{abstract}

Keywords: Smiles rearrangement, diarylamines, phenol, amine, synthesis

\section{Introduction}

Diarylamines represent an important class of compounds due to their wide applications and special pharmacological activities. ${ }^{1,2}$ Therefore, a facile and reliable access to diarylamine derivatives is of great importance. Among numerous methods to prepare diarylamines, the use of metallic catalysts, especially $\mathrm{Pd}_{2}(\mathrm{dba})_{3}$ and $\mathrm{Pd}(\mathrm{OAc})_{2}$, is one of the most attractive routes. ${ }^{3-10}$ These metals catalyze the reaction only in the form of metal-organic complexes, which constitute the active catalysts in many $\mathrm{C}-\mathrm{N}$ bond formation cross-coupling methodologies for the synthesis of diarylamines, while such complexes are quite difficult to prepare and extremely air sensitive. ${ }^{11}$ Although the reactions can sometimes be catalyzed by diverse ligands, the different kind and concentration of ligands may lead to various products. Accordingly, the amount of ligands must be selected carefully. ${ }^{12}$

On the other hand, Wolfe et al. ${ }^{13}$ developed a general procedure for the Pd-catalyzed intermolecular amination which proceeds at ambient temperatures, but only aryl iodides can react with a few substituted arylamines in the presence of a strong base like $\mathrm{NaO} t$-Bu. An alternative method of $\mathrm{C}-\mathrm{N}$ bond formation for the preparation of $\mathrm{N}$-arylamines is the Smiles rearrangement. Solid-liquid phase-transfer catalyst tris(3,6-dioxaheptylamine) (TDA-1) activates $N$-alkylation of chlorinated phenoxyacetamides in the presence of $\mathrm{KOH}$ via Smiles rearrangement; however, the 
substrates are limited to trichlorophenol. ${ }^{14}$ Substituted aryloxyacetamides with oxygen and nitrogen separated by the $\mathrm{CH}_{2} \mathrm{CO}$ group undergo Smiles rearrangement to give $\mathrm{N}$-alkyl- and $N$ arylamines under $\mathrm{EtONa} / \mathrm{EtOH}$ or $\mathrm{NaH} / \mathrm{DMF}$ conditions, while only aryloxy rings containing only a weak or no electron-withdrawing group proceed in such a rearrangement. ${ }^{15}$ Acemoglu found that the major side reactions to form $N$-arylamines can be avoided by using $\mathrm{K}_{2} \mathrm{CO}_{3} / i-\mathrm{PrOH}$ system followed by $\mathrm{MeONa} / \mathrm{MeOH} .{ }^{16}$ It was also found that a Smiles rearrangement is involved in the preparation of nitro group containing alkylarylamines, ${ }^{17}$ and $\mathrm{N}$-arylamines. ${ }^{18}$

\section{Results and Discussion}

Our interest in reactions via Smiles rearrangement with two or more reagents being used simultaneously led us to use substituted 2-chlorophenols and arylamines, activated by chloroacetyl chloride for $\mathrm{C}-\mathrm{N}$ bond formation to obtain diarylamines and arylalkylamines. ${ }^{19}$ The limitation of our methodology is that all phenols contain a chlorine atom in ortho position of the phenolic hydroxyl group. This work, combined with our Smiles rearrangement reaction system has recently led us to design the reaction of diverse phenols, amines and chloroacetyl chloride to prepare diarylamines, with chloroacetyl chloride activating these reactions (Scheme 1).

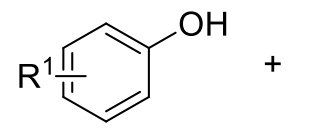

1<smiles>O=C(Cl)CCl</smiles> 
nitroaniline $\mathbf{2 g}$, the yield was lower (65\%). In other cases, $m$-cresol $\mathbf{1 a}$ and $p$-cresol $\mathbf{1 b}$ reacted with anilines $\mathbf{2 a}-\mathbf{f}$ to give the corresponding diarylamines $\mathbf{3 a}-\mathbf{k}$ after a longer reaction time (60$80 \mathrm{~min})$. Considering a stronger base may shorten the reaction time of the Smiles rearrangement, ${ }^{21} \mathrm{NaOH}$ was employed, but no products were found under this condition.

Table 1. One-pot synthesis of diarylamine 3

\begin{tabular}{|c|c|c|c|c|c|c|c|}
\hline Entry & Phenol & & Amine & & Diarylamine & & Yield $\%^{\mathrm{a}}$ \\
\hline 1 & & $1 \mathbf{a}$ & $-\mathrm{OCH}_{3}$ & $2 \mathbf{a}$ & & $3 \mathbf{a}$ & 77 \\
\hline 2 & & $1 \mathbf{a}$ & & $2 \mathrm{~b}$ & & $3 \mathbf{b}$ & 90 \\
\hline 3 & & $1 \mathbf{a}$ & & $2 c$ & & $3 c$ & 67 \\
\hline 4 & & $1 \mathbf{a}$ & & $2 d$ & & 3d & 81 \\
\hline 5 & & $1 \mathbf{a}$ & $-\mathrm{NO}_{2}$ & $2 e$ & & $3 \mathbf{e}$ & 65 \\
\hline 6 & $-c$ & $1 b$ & $-\mathrm{OCH}_{3}$ & $\mathbf{2 a}$ & & $3 f$ & 80 \\
\hline 7 & $-c$ & $1 b$ & & $2 \mathrm{~b}$ & & $3 g$ & 90 \\
\hline 8 & $-c$ & 1b & & $2 c$ & & $3 \mathbf{h}$ & 63 \\
\hline 9 & & $1 b$ & & 2d & & $3 \mathbf{i}$ & 68 \\
\hline 10 & 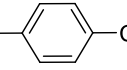 & $1 b$ & & $2 e$ & & $\mathbf{3 j}$ & 58 \\
\hline 11 & & $1 \mathrm{~b}$ & $-\mathrm{CH}_{3}$ & $2 f$ & & $3 \mathbf{k}$ & 74 \\
\hline 12 & & 1c & $-\mathrm{OCH}_{3}$ & $2 \mathbf{a}$ & & 31 & 75 \\
\hline 13 & & 1c & & $2 b$ & & $3 m$ & 88 \\
\hline
\end{tabular}


Table 1. Continued

Entry

${ }^{a}$ Yield of isolated product. Reaction conditions: phenols $(1.0 \mathrm{mmol})$, amines $(1.0 \mathrm{mmol})$, chloroacetyl chloride $(1.2 \mathrm{mmol}), \mathrm{Cs}_{2} \mathrm{CO}_{3}(3.2 \mathrm{mmol})$, DMF $(25 \mathrm{~mL}), 150^{\circ} \mathrm{C}, \mathrm{MW}$ irradiation.

A hypothesized explanation for the results is given in Scheme 2. The O-alkylated phenol 4 undergoes Smiles rearrangement by nucleophilic attack of the nitrogen at the benzene ring carbon atom attached to oxygen forming a new $\mathrm{C}-\mathrm{N}$ bond to give spiro-intermediate $\mathbf{5}$. Rearomatization and opening of the oxazolidinone ring in $\mathbf{5}$ followed by alkaline hydrolysis of the resulting intermediate 6 in the presence of $\mathrm{Cs}_{2} \mathrm{CO}_{3}$ affords the diarylamine derivative 3 . As shown by the reactivity of examples mentioned above, the intramolecular nucleophilic aromatic substitution of $\mathbf{4}$ forming intermediate $\mathbf{5}$ is favored by electron-donating groups $\mathrm{R}^{2}$ in the aniline ring and by electron-withdrawing groups $\mathrm{R}^{1}$ in the phenol ring.

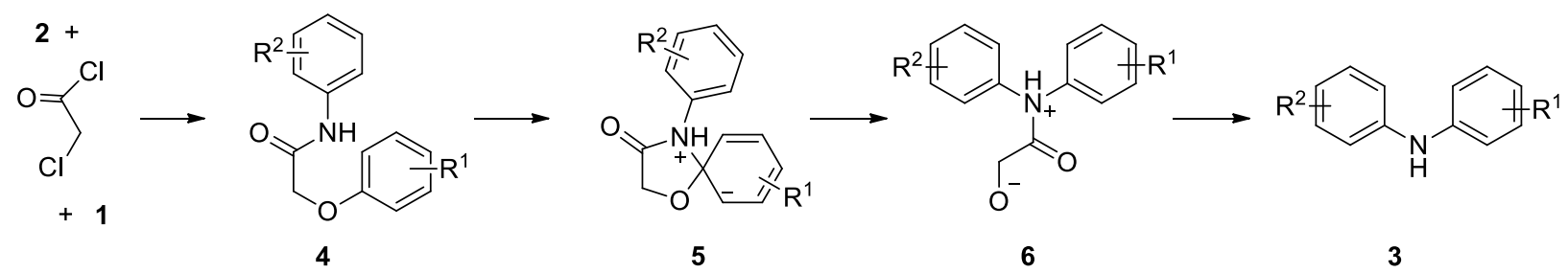

Scheme 2. Proposed mechanism for the formation of diarylamines $\mathbf{3}$ via Smiles rearrangement. 


\section{Conclusions}

In summary, various diarylamines $\mathbf{3}$ were effectively synthesized starting from readily available substituted phenols, anilines, and chloroacetyl chloride (1.0:1.0:1.2 equiv.) via Smiles rearrangement. Mechanistic studies of this method, as well as further applications in the preparation of biologically interesting compounds are under active investigation in our laboratory.

\section{Experimental Section}

General. ${ }^{1} \mathrm{H}$ and ${ }^{13} \mathrm{C}$ NMR spectra (at $300 \mathrm{MHz}$ and $75 \mathrm{MHz}$, respectively) were recorded in $\mathrm{CDCl}_{3}$ with tetramethylsilane as internal reference on a Bruker Advance 300 FT spectrometer. Chemical shifts were reported in parts per million. Mass spectra (MS) were measured by ESI. $\mathrm{CDCl}_{3}$ was used as delivered from Sigma-Aldrich. Silica gel (70-230 mesh) was used for flash column chromatography. All reactions were monitored by TLC using $0.25 \mathrm{~mm}$ silica gel plates with UV indicator (Merck 60F254). The microwave-assisted reaction time is the hold time at the final temperature. Unless otherwise noted, other reagents were obtained from commercial suppliers and used without further purification. Microwave XH-100B (made in Beijing XiangHu Science and Technology Development Co., LTD, P.R. China) was used to carry out the reactions.

\section{Smiles rearrangement for the synthesis of diarylamines (3). General procedure}

To a magnetically stirred solution of arylamine $2(1.0 \mathrm{mmol})$ and $\mathrm{Cs}_{2} \mathrm{CO}_{3}(1042.6 \mathrm{mg}, 3.2 \mathrm{mmol})$ in dry DMF, cooled in an ice bath, were added chloroacetyl chloride $(135.5 \mathrm{mg}, 1.2 \mathrm{mmol})$ and substituted phenol $1(1.0 \mathrm{mmol})$. The reaction mixture was stirred for $30 \mathrm{~min}$ at room temperature, then placed in a microwave oven $\left(600 \mathrm{~W}, 150{ }^{\circ} \mathrm{C}\right)$ and irradiated for 30-80 min. The solvent was removed under vacuum, and water $(20 \mathrm{~mL})$ was added into the residue. The mixture was then extracted with ethyl acetate $(4 \times 30 \mathrm{~mL})$. The organic layers were combined, dried over anhydrous $\mathrm{MgSO}_{4}$, and evaporated under vacuum to give the crude product 3 . The pure product 3 was obtained by column chromatography on silica gel.

$\mathrm{N}$-(4-Methoxyphenyl)-3-methylaniline (3a). ${ }^{22}$ Gray solid; mp 72-75 ${ }^{\circ} \mathrm{C}$ (lit. ${ }^{22} 75-76{ }^{\circ} \mathrm{C}$ ). ${ }^{1} \mathrm{H}$ NMR $\left(300 \mathrm{MHz}, \mathrm{CDCl}_{3}\right): \delta 2.28\left(\mathrm{~s}, 3 \mathrm{H} ; \mathrm{CH}_{3}\right), 3.80\left(\mathrm{~s}, 3 \mathrm{H} ; \mathrm{OCH}_{3}\right), 5.45$ (s, br, $\left.1 \mathrm{H} ; \mathrm{NH}\right), 6.64-$ $7.24\left(\mathrm{~m}, 8 \mathrm{H} ; \mathrm{H}_{\mathrm{Ar}}\right) .{ }^{13} \mathrm{C} \mathrm{NMR}\left(75 \mathrm{MHz}, \mathrm{CDCl}_{3}\right): \delta 21.5\left(\mathrm{CH}_{3}\right), 55.6\left(\mathrm{OCH}_{3}\right), 112.8(\mathrm{CH}), 114.6$ $(\mathrm{CH}), 116.3(\mathrm{CH}), 120.5(\mathrm{CH}), 122.2(\mathrm{CH}), 129.1(\mathrm{CH}), 135.9(\mathrm{C}), 139.1(\mathrm{C}), 145.1(\mathrm{C}), 155.2$ (C).

3-Methyl- $N$-phenylaniline (3b). ${ }^{23}$ Yellow liquid. ${ }^{1} \mathrm{H}$ NMR $\left(300 \mathrm{MHz}, \mathrm{CDCl}_{3}\right)$ : $\delta 2.29$ (s, 3H; $\left.\mathrm{CH}_{3}\right), 5.58(\mathrm{~s}, 1 \mathrm{H} ; \mathrm{NH}), 6.85-7.28\left(\mathrm{~m}, 9 \mathrm{H} ; \mathrm{H}_{\mathrm{Ar}}\right) .{ }^{13} \mathrm{C} \mathrm{NMR}\left(75 \mathrm{MHz}, \mathrm{CDCl}_{3}\right): \delta 20.7\left(\mathrm{CH}_{3}\right)$, $116.8(\mathrm{CH}), 117.8(\mathrm{CH}), 118.9(\mathrm{CH}), 120.2(\mathrm{CH}), 120.9(\mathrm{CH}), 129.3(\mathrm{CH}), 129.8(\mathrm{CH}), 130.9$ $(\mathrm{CH}), 140.2(\mathrm{C}), 143.9(\mathrm{C})$. 
2-Methyl- $\boldsymbol{N}$-( $\boldsymbol{m}$-tolyl)aniline (3c). ${ }^{24}$ Yellow liquid. ${ }^{1} \mathrm{H}$ NMR $\left(300 \mathrm{MHz}, \mathrm{CDCl}_{3}\right)$ : $\delta 2.22$ (s, 3H; $\left.\mathrm{CH}_{3}\right), 2.28\left(\mathrm{~s}, 3 \mathrm{H} ; \mathrm{CH}_{3}\right), 5.29(\mathrm{~s}, \mathrm{br}, 1 \mathrm{H} ; \mathrm{NH}), 6.69-7.23\left(\mathrm{~m}, 8 \mathrm{H} ; \mathrm{H}_{\mathrm{Ar}}\right) .{ }^{13} \mathrm{C} \mathrm{NMR}(75 \mathrm{MHz}$, $\left.\mathrm{CDCl}_{3}\right): \delta 17.8\left(\mathrm{CH}_{3}\right), 21.45\left(\mathrm{CH}_{3}\right), 114.5(\mathrm{CH}), 118.1(\mathrm{CH}), 118.8(\mathrm{CH}), 121.3(\mathrm{CH}), 121.8$ $(\mathrm{CH}), 126.7(\mathrm{CH}), 128.1(\mathrm{C}), 129.1(\mathrm{CH}), 130.8(\mathrm{CH}), 139.1(\mathrm{C}), 141.2(\mathrm{C}), 143.8(\mathrm{C})$.

Di-(m-tolyl)amine (3d). ${ }^{25}$ Colorless liquid. ${ }^{1} \mathrm{H} \mathrm{NMR}\left(300 \mathrm{MHz}, \mathrm{CDCl}_{3}\right): \delta 2.25\left(\mathrm{~s}, 6 \mathrm{H} ; \mathrm{CH}_{3}\right)$, 5.46 (s, br, 1H; NH), 6.69 (d, J = 7.2 Hz, 2H; HAr), 6.79-6.83 (m, 4H; $\left.\mathrm{H}_{\mathrm{Ar}}\right), 7.07-7.12$ (m, 2H; $\left.\mathrm{H}_{\mathrm{Ar}}\right) .{ }^{13} \mathrm{C} \mathrm{NMR}\left(75 \mathrm{MHz}, \mathrm{CDCl}_{3}\right): \delta 21.4\left(\mathrm{CH}_{3}\right), 114.8(\mathrm{CH}), 118.5(\mathrm{CH}), 121.6(\mathrm{CH}), 129.0$ (CH), 139.0 (C), $143.1(\mathrm{C})$.

3-Methyl- $\boldsymbol{N}$-(4-nitrophenyl)aniline (3e). Brown solid; mp 130-133 ${ }^{\circ} \mathrm{C} . R_{\mathrm{f}}=0.45$ (petroleum ether/AcOEt, 6:1). ${ }^{1} \mathrm{H}$ NMR (300 MHz, $\mathrm{CDCl}_{3}$ ): $\delta 2.37$ (s, 3H; $\mathrm{CH}_{3}$ ), 6.29 (s, br, 1H; $\left.\mathrm{NH}\right), 6.94$, 6.91 (AA'; 2H; $\left.\mathrm{H}_{\mathrm{Ar}}\right), 6.97-7.02\left(\mathrm{~m}, 3 \mathrm{H} ; \mathrm{H}_{\mathrm{Ar}}\right), 7.25-7.27\left(\mathrm{~m}, 1 \mathrm{H}, \mathrm{H}_{\mathrm{Ar}}\right), 8.13,8.10\left(\mathrm{XX}^{\prime} ; 2 \mathrm{H}\right.$; $\left.\mathrm{H}_{\mathrm{Ar}}\right) .{ }^{13} \mathrm{C} \mathrm{NMR}\left(75 \mathrm{MHz}, \mathrm{CDCl}_{3}\right): \delta 21.4\left(\mathrm{CH}_{3}\right), 113.6(\mathrm{CH}), 119.0(\mathrm{CH}), 122.6(\mathrm{CH}), 125.5$ $(\mathrm{CH}), 126.2(\mathrm{CH}), 129.5(\mathrm{C}), 139.4(\mathrm{C}), 139.6(\mathrm{C}), 139.7(\mathrm{C}), 150.3(\mathrm{C}) . \mathrm{MS}(\mathrm{ESI}): \mathrm{m} / z(\%) 229$ (8) $[\mathrm{M}+1], 212$ (57), 182 (74), 167 (100). Anal. Calcd for $\mathrm{C}_{13} \mathrm{H}_{12} \mathrm{~N}_{2} \mathrm{O}_{2}$ : C, 68.40; H, 5.30; N, 12.27. Found: C, 68.64; H, 5.66; N, 11.98 .

4-Methoxy- $\boldsymbol{N}$-(p-tolyl)aniline (3f). ${ }^{20}$ Gray solid; mp 80-82 ${ }^{\circ} \mathrm{C}\left(\right.$ lit. $\left.^{20} 82{ }^{\circ} \mathrm{C}\right) .{ }^{1} \mathrm{H} \mathrm{NMR}(300 \mathrm{MHz}$, $\left.\mathrm{CDCl}_{3}\right): \delta 2.27\left(\mathrm{~s}, 3 \mathrm{H} ; \mathrm{CH}_{3}\right), 3.78\left(\mathrm{~s}, 3 \mathrm{H} ; \mathrm{OCH}_{3}\right), 5.38(\mathrm{~s}, \mathrm{br}, 1 \mathrm{H} ; \mathrm{NH}), 6.83-6.85\left(\mathrm{~m}, 4 \mathrm{H} ; \mathrm{H}_{\mathrm{Ar}}\right)$, 7.00-7.04 (m, 4H; $\left.\mathrm{H}_{\mathrm{Ar}}\right) .{ }^{13} \mathrm{C} \mathrm{NMR}\left(75 \mathrm{MHz}, \mathrm{CDCl}_{3}\right): \delta 20.5\left(\mathrm{CH}_{3}\right), 55.6\left(\mathrm{OCH}_{3}\right), 114.6(\mathrm{CH})$, $116.5(\mathrm{CH}), 121.1(\mathrm{CH}), 129.3(\mathrm{C}), 129.8(\mathrm{CH}), 136.6(\mathrm{C}), 142.4(\mathrm{C}), 154.7(\mathrm{C})$.

4-Methyl- $\boldsymbol{N}$-phenylaniline (3g). ${ }^{26}$ White solid; mp 85-87 ${ }^{\circ} \mathrm{C}\left(\right.$ lit. $\left.^{26} 87^{\circ} \mathrm{C}\right) .{ }^{1} \mathrm{H} \mathrm{NMR}(300 \mathrm{MHz}$, $\left.\mathrm{CDCl}_{3}\right): \delta 2.24\left(\mathrm{~s}, 3 \mathrm{H} ; \mathrm{CH}_{3}\right), 5.49(\mathrm{~s}, \mathrm{br}, 1 \mathrm{H} ; \mathrm{NH}), 6.54-7.22\left(\mathrm{~m}, 9 \mathrm{H} ; \mathrm{H}_{\mathrm{Ar}}\right)$.

2-Methyl- $\boldsymbol{N}$-(p-tolyl)aniline (3h). ${ }^{27}$ Yellow viscous oil. ${ }^{1} \mathrm{H} \mathrm{NMR}\left(300 \mathrm{MHz}, \mathrm{CDCl}_{3}\right): \delta 2.25$ (s, $\left.3 \mathrm{H} ; \mathrm{CH}_{3}\right), 2.30$ (s, 3H; $\left.\mathrm{CH}_{3}\right), 5.29$ (s, br, 1H; NH), 6.85-6.95 (m, 3H; $\mathrm{H}_{\mathrm{Ar}}$ ), 7.06-7.24 (m, 5H; $\left.\mathrm{H}_{\mathrm{Ar}}\right) .{ }^{13} \mathrm{C} \mathrm{NMR}\left(75 \mathrm{MHz}, \mathrm{CDCl}_{3}\right): \delta 17.8\left(\mathrm{CH}_{3}\right), 20.6\left(\mathrm{CH}_{3}\right), 117.2(\mathrm{CH}), 118.6(\mathrm{CH}), 121.0$ $(\mathrm{CH}), 126.7(\mathrm{CH}), 126.9(\mathrm{C}), 129.8(\mathrm{CH}), 130.4(\mathrm{C}), 130.8(\mathrm{CH}), 141.0(\mathrm{C}), 142.0(\mathrm{C})$.

3-Methyl- $\boldsymbol{N}$-(p-tolyl)aniline (3i). ${ }^{28}$ Yellow viscous oil. ${ }^{1} \mathrm{H}$ NMR $\left(300 \mathrm{MHz}, \mathrm{CDCl}_{3}\right): \delta 2.27$ (s, $3 \mathrm{H} ; \mathrm{CH}_{3}$ ), 2.28 (s, 3H; $\mathrm{CH}_{3}$ ), 5.49 (s, br, $\left.1 \mathrm{H} ; \mathrm{NH}\right), 6.68$ (d, J=7.2 Hz, 1H; $\mathrm{H}_{\mathrm{Ar}}$ ), 6.80-7.21 (m, $\left.7 \mathrm{H} ; \mathrm{H}_{\mathrm{Ar}}\right) .{ }^{13} \mathrm{C} \mathrm{NMR}\left(75 \mathrm{MHz}, \mathrm{CDCl}_{3}\right): \delta 20.6\left(\mathrm{CH}_{3}\right), 21.5\left(\mathrm{CH}_{3}\right), 113.9(\mathrm{CH}), 117.5(\mathrm{CH}), 118.8$ $(\mathrm{CH}), 121.1(\mathrm{CH}), 129.1(\mathrm{CH}), 129.8(\mathrm{CH}), 130.7(\mathrm{C}), 139.1(\mathrm{C}), 140.3(\mathrm{C}), 143.8(\mathrm{C})$.

4-Methyl- $\boldsymbol{N}$-(4-nitrophenyl)aniline (3j). ${ }^{29}$ Brown solid; mp $132-135{ }^{\circ} \mathrm{C}$ (lit. ${ }^{29} 137{ }^{\circ} \mathrm{C}$ ). ${ }^{1} \mathrm{H}$ NMR (300 MHz, $\left.\mathrm{CDCl}_{3}\right): \delta 2.36\left(\mathrm{~s}, 6 \mathrm{H} ; \mathrm{CH}_{3}\right), 6.29$ (s, br, 1H; NH), 6.88, $6.85\left(\mathrm{AA}^{\prime} ; 2 \mathrm{H} ; \mathrm{H}_{\mathrm{Ar}}\right.$ ), 7.12, 7.09 (AA'; 2H; $\left.\mathrm{H}_{\mathrm{Ar}}\right), 7.21,7.18\left(\mathrm{BB}^{\prime} ; 2 \mathrm{H} ; \mathrm{H}_{\mathrm{Ar}}\right), 8.10,8.07\left(\mathrm{XX}^{\prime} ; 2 \mathrm{H} ; \mathrm{H}_{\mathrm{Ar}}\right) .{ }^{13} \mathrm{C} \mathrm{NMR}(75$ $\left.\mathrm{MHz}, \mathrm{CDCl}_{3}\right): \delta 20.9\left(\mathrm{CH}_{3}\right), 113.1(\mathrm{CH}), 122.6(\mathrm{CH}), 126.2(\mathrm{CH}), 130.2(\mathrm{CH}), 134.8(\mathrm{C}), 136.6$ (C), $139.2(\mathrm{C}), 150.8(\mathrm{C})$.

Di-(p-tolyl)amine (3k). ${ }^{30}$ Light yellow solid; mp 74-77 ${ }^{\circ} \mathrm{C}$ (lit. $\left.{ }^{30} 73-75{ }^{\circ} \mathrm{C}\right) .{ }^{1} \mathrm{H}$ NMR $(300$ $\left.\mathrm{MHz}_{\mathrm{CDCl}}\right): \delta 2.28\left(\mathrm{~s}, 6 \mathrm{H} ; \mathrm{CH}_{3}\right), 5.49(\mathrm{~s}, \mathrm{br}, 1 \mathrm{H} ; \mathrm{NH}), 6.95,6.92\left(\mathrm{AA}^{\prime} ; 4 \mathrm{H} ; \mathrm{H}_{\mathrm{Ar}}\right), 7.07,7.04$ $\left(\mathrm{BB}^{\prime} ; 4 \mathrm{H} ; \mathrm{H}_{\mathrm{Ar}}\right) .{ }^{13} \mathrm{C} \mathrm{NMR}\left(75 \mathrm{MHz}, \mathrm{CDCl}_{3}\right): \delta 20.6\left(\mathrm{CH}_{3}\right), 117.9(\mathrm{CH}), 129.8(\mathrm{CH}), 130.1(\mathrm{C})$, $141.1(\mathrm{C})$.

4-Methoxy- $\boldsymbol{N}$-(4-nitrophenyl)aniline (31). ${ }^{31}$ Brown solid; mp 149-152 ${ }^{\circ} \mathrm{C}$ (lit. $\left.{ }^{31} 152-153{ }^{\circ} \mathrm{C}\right)$. ${ }^{1} \mathrm{H}$ NMR $\left(300 \mathrm{MHz}, \mathrm{CDCl}_{3}\right): \delta 3.84\left(\mathrm{~s}, 3 \mathrm{H} ; \mathrm{OCH}_{3}\right), 6.16(\mathrm{~s}, 1 \mathrm{H} ; \mathrm{NH}), 6.78,6.75\left(\mathrm{AA}^{\prime} ; 2 \mathrm{H} ; \mathrm{H}_{\mathrm{Ar}}\right)$, 
6.95, $6.92\left(\mathrm{AA}^{\prime} ; 2 \mathrm{H} ; \mathrm{H}_{\mathrm{Ar}}\right), 7.17,7.14\left(\mathrm{BB}^{\prime} ; 2 \mathrm{H} ; \mathrm{H}_{\mathrm{Ar}}\right), 8.10,8.07\left(\mathrm{XX} ; 2 \mathrm{H} ; \mathrm{H}_{\mathrm{Ar}}\right) .{ }^{13} \mathrm{C}$ NMR $(75$ $\left.\mathrm{MHz}, \mathrm{CDCl}_{3}\right): \delta 55.5\left(\mathrm{OCH}_{3}\right), 112.6(\mathrm{CH}), 114.9(\mathrm{CH}), 125.5(\mathrm{CH}), 126.3(\mathrm{CH}), 131.9(\mathrm{C})$, 139.0 (C), 151.7 (C), 157.4 (C).

4-Nitro- $N$-phenylaniline (3m). ${ }^{32}$ Brown solid; mp 131-133 ${ }^{\circ} \mathrm{C}$ (lit. $\left.{ }^{32} 131{ }^{\circ} \mathrm{C}\right) .{ }^{1} \mathrm{H}$ NMR $(300$ $\left.\mathrm{MHz}, \mathrm{CDCl}_{3}\right): \delta 6.40(\mathrm{~s}, 1 \mathrm{H} ; \mathrm{NH}), 6.96,6.93\left(\mathrm{AA}^{\prime} ; 2 \mathrm{H} ; \mathrm{H}_{\mathrm{Ar}}\right), 7.14-7.42\left(\mathrm{~m}, 5 \mathrm{H} ; \mathrm{H}_{\mathrm{Ar}}\right), 8.13,8.10$ $\left(\mathrm{XX} ; 2 \mathrm{H} ; \mathrm{H}_{\mathrm{Ar} .}{ }^{13} \mathrm{C}\right.$ NMR $\left(75 \mathrm{MHz}, \mathrm{CDCl}_{3}\right): \delta 113.6(\mathrm{CH}), 121.9(\mathrm{CH}), 124.6(\mathrm{C}), 126.2(\mathrm{CH})$, 129.7(CH), $139.4(\mathrm{C}), 139.6(\mathrm{C}), 150.2(\mathrm{C})$.

2-Methyl- $N$-(4-nitrophenyl)aniline (3n). Brown solid; $\mathrm{mp} 130-133{ }^{\circ} \mathrm{C} . R_{\mathrm{f}}=0.48$ (petroleum ether/AcOEt, 6:1). ${ }^{1} \mathrm{H}$ NMR (300 MHz, $\left.\mathrm{CDCl}_{3}\right): \delta 2.25$ (s, 3H; $\left.\mathrm{CH}_{3}\right), 6.10(\mathrm{~s}, 1 \mathrm{H} ; \mathrm{NH}), 6.73$, $6.70\left(\mathrm{AA}^{\prime} ; 2 \mathrm{H} ; \mathrm{H}_{\mathrm{Ar}}\right), 7.18-7.31\left(\mathrm{~m}, 4 \mathrm{H} ; \mathrm{H}_{\mathrm{Ar}}\right), 8.10,8.07$ (XX'; $\left.2 \mathrm{H} ; \mathrm{H}_{\mathrm{Ar}}\right) .{ }^{13} \mathrm{C}$ NMR $(75 \mathrm{MHz}$, $\left.\mathrm{CDCl}_{3}\right): \delta 17.8\left(\mathrm{CH}_{3}\right), 113.0(\mathrm{CH}), 124.7(\mathrm{CH}), 126.1(\mathrm{CH}), 126.2(\mathrm{CH}), 127.1(\mathrm{CH}), 131.4$ (CH), 133.2 (C), 137.5 (C), 139.1 (C), 151.3 (C). MS (ESI): m/z (\%) 229 (9) [M+1], 212 (100), 182 (77), 168 (94). Anal. Calcd for $\mathrm{C}_{13} \mathrm{H}_{12} \mathrm{~N}_{2} \mathrm{O}_{2}: \mathrm{C}, 68.40 ; \mathrm{H}, 5.30 ; \mathrm{N}, 12.27$. Found: $\mathrm{C}, 68.40 ; \mathrm{H}$, $5.43 ; \mathrm{N}, 12.36$.

4-(4-Methoxyphenylamino)benzaldehyde (3o). ${ }^{33}$ Brown solid; mp 108-111 ${ }^{\circ} \mathrm{C}\left(\right.$ lit. $^{33} 113{ }^{\circ} \mathrm{C}$ ). ${ }^{1} \mathrm{H}$ NMR (300 MHz, $\left.\mathrm{CDCl}_{3}\right): \delta 3.83\left(\mathrm{~s}, 3 \mathrm{H} ; \mathrm{OCH}_{3}\right), 6.08(\mathrm{~s}, 1 \mathrm{H} ; \mathrm{NH}), 6.87,6.84\left(\mathrm{AA}^{\prime} ; 2 \mathrm{H} ; \mathrm{H}_{\mathrm{Ar}}\right.$ ), 6.94, $6.91\left(\mathrm{AA}^{\prime} ; 2 \mathrm{H} ; \mathrm{H}_{\mathrm{Ar}}\right), 7.17,7,14\left(\mathrm{BB}^{\prime} ; 2 \mathrm{H} ; \mathrm{H}_{\mathrm{Ar}}\right), 7.72,7.69\left(\mathrm{XX}^{\prime} ; 2 \mathrm{H} ; \mathrm{H}_{\mathrm{Ar}}\right), 9.75(\mathrm{~s}, 1 \mathrm{H}$; $\mathrm{CHO}) .{ }^{13} \mathrm{C}$ NMR $\left(75 \mathrm{MHz}, \mathrm{CDCl}_{3}\right): \delta 55.5\left(\mathrm{OCH}_{3}\right), 113.4(\mathrm{CH}), 114.8(\mathrm{CH}), 125.1(\mathrm{CH}), 127.8$ (C), $132.2(\mathrm{CH}), 132.6(\mathrm{C}), 151.4(\mathrm{C}), 157.0(\mathrm{C}), 190.2(\mathrm{CHO})$.

4-(Phenylamino)benzaldehyde (3p)..$^{34}$ Brown solid; mp 94-97 ${ }^{\circ} \mathrm{C}$ (lit. ${ }^{34} 95-97{ }^{\circ} \mathrm{C}$ ). ${ }^{1} \mathrm{H}$ NMR $\left(300 \mathrm{MHz}, \mathrm{CDCl}_{3}\right): \delta 6.38(\mathrm{~s}, 1 \mathrm{H} ; \mathrm{NH}), 7.04,7.01\left(\mathrm{AA}^{\prime} ; 2 \mathrm{H} ; \mathrm{H}_{\mathrm{Ar}}\right), 7.09-7.39\left(\mathrm{~m}, 5 \mathrm{H} ; \mathrm{H}_{\mathrm{Ar}}\right), 7.75$, $7.72\left(\mathrm{XX}^{\prime} ; 2 \mathrm{H} ; \mathrm{H}_{\mathrm{Ar}}\right), 9.78(\mathrm{~s}, 1 \mathrm{H} ; \mathrm{CHO}) .{ }^{13} \mathrm{C} \mathrm{NMR}\left(75 \mathrm{MHz}, \mathrm{CDCl}_{3}\right): \delta 114.4(\mathrm{CH}), 121.3(\mathrm{CH})$, $123.8(\mathrm{CH}), 128.4(\mathrm{C}), 129.5(\mathrm{CH}), 132.1(\mathrm{CH}), 140.0(\mathrm{C}), 149.8(\mathrm{C}), 190.4(\mathrm{CHO})$.

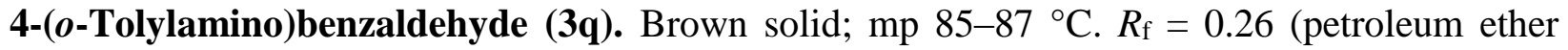
/AcOEt, 6:1). ${ }^{1} \mathrm{H}$ NMR $\left(300 \mathrm{MHz}, \mathrm{CDCl}_{3}\right): \delta 2.25\left(\mathrm{~s}, 3 \mathrm{H} ; \mathrm{CH}_{3}\right), 6.00(\mathrm{~s}, 1 \mathrm{H} ; \mathrm{NH}), 6.83,6.80$ (AA'; 2H; $\mathrm{H}_{\mathrm{Ar}}$ ), 7.13-7.27 (m, 4H; $\left.\mathrm{H}_{\mathrm{Ar}}\right), 7.73,7.70\left(\mathrm{XX} ; 2 \mathrm{H} ; \mathrm{H}_{\mathrm{Ar}}\right), 9.76(\mathrm{~s}, 1 \mathrm{H} ; \mathrm{CHO}) .{ }^{13} \mathrm{C}$ NMR (75 MHz, $\left.\mathrm{CDCl}_{3}\right): \delta 17.9\left(\mathrm{CH}_{3}\right), 113.8(\mathrm{CH}), 124.1(\mathrm{CH}), 125.4(\mathrm{CH}), 127.0(\mathrm{CH}), 127.9$ (C), $131.3(\mathrm{CH}), 132.1(\mathrm{CH}), 132.6(\mathrm{C}), 138.0(\mathrm{C}), 151.0(\mathrm{C}), 190.3(\mathrm{CHO})$. MS (ESI): $\mathrm{m} / \mathrm{z}(\%)$ 212 (28) [M+1], 184 (12), 183 (9), 182 (14), 169 (52), 168 (24), 103 (48), 88 (100), 75 (11), 60 (10). Anal. Calcd for $\mathrm{C}_{14} \mathrm{H}_{13} \mathrm{NO}$ : C, 79.60; H, 6.20; N, 6.63. Found: C, 79.42; H, 6.32; N, 6.66.

4-(4-(m-Tolylamino)benzaldehyde (3r). Brown solid; mp $117-119{ }^{\circ} \mathrm{C} . R_{\mathrm{f}}=0.23$ (petroleum ether/AcOEt, 6:1). ${ }^{1} \mathrm{H}$ NMR (300 MHz, $\left.\mathrm{CDCl}_{3}\right): \delta 2.34$ (s, 3H; $\left.\mathrm{CH}_{3}\right), 6.37(\mathrm{~s}, 1 \mathrm{H} ; \mathrm{NH}), 6.92-$ $7.24\left(\mathrm{~m}, 6 \mathrm{H} ; \mathrm{H}_{\mathrm{Ar}}\right), 7.74,7.71\left(\mathrm{AA}^{\prime}, 2 \mathrm{H} ; \mathrm{H}_{\mathrm{Ar}}\right), 9.77(\mathrm{~s}, 1 \mathrm{H} ; \mathrm{CHO}) .{ }^{13} \mathrm{C} \mathrm{NMR}\left(75 \mathrm{MHz}, \mathrm{CDCl}_{3}\right): \delta$ $21.4\left(\mathrm{CH}_{3}\right), 114.4(\mathrm{CH}), 118.3(\mathrm{CH}), 121.9(\mathrm{CH}), 124.7(\mathrm{CH}), 128.2(\mathrm{C}), 129.3(\mathrm{CH}), 132.1$ (CH), 139.5 (C), 139.9 (C), 150.0 (C), 190.4 (CHO). MS (ESI): m/z (\%) 212 (33) [M+1], 183 (21), 169 (69), 103 (81), 88 (100), 75 (34), 73 (6), 60 (21). Anal. Calcd for $\mathrm{C}_{14} \mathrm{H}_{13} \mathrm{NO}$ : C, 79.59 ; H, 6.20; N, 6.63. Found: C, 79.20; H, 6.35; N, 6.61.

4-( $p$-Tolylamino)benzaldehyde (3s). Brown solid; $\mathrm{mp} 85-88{ }^{\circ} \mathrm{C} . R_{\mathrm{f}}=0.20$ (petroleum ether/ AcOEt, 6:1). ${ }^{1} \mathrm{H}$ NMR (300 MHz, $\left.\mathrm{CDCl}_{3}\right): \delta 2.35$ (s, 3H; $\left.\mathrm{CH}_{3}\right), 6.20(\mathrm{~s}, 1 \mathrm{H} ; \mathrm{NH}), 6.97,6.94$ (AA'; 2H; $\mathrm{H}_{\mathrm{Ar}}$ ), 7.12, 7.09 (AA'; 2H; $\mathrm{H}_{\mathrm{Ar}}$ ), 7.19, 7.16 (BB'; 2H; $\mathrm{H}_{\mathrm{Ar}}$ ), 7.73, $7.70\left(\mathrm{XX} ; 2 \mathrm{H} ; \mathrm{H}_{\mathrm{Ar}}\right.$ ), 
9.77 (s, 1H; CHO). ${ }^{13} \mathrm{C}$ NMR (75 MHz, $\left.\mathrm{CDCl}_{3}\right): \delta 20.8\left(\mathrm{CH}_{3}\right), 113.9(\mathrm{CH}), 122.1(\mathrm{CH}), 128.1$ (C), $130.1(\mathrm{CH}), 132.1(\mathrm{CH}), 134.0(\mathrm{C}), 137.2$ (C), 150.5 (C), 190.3 (CHO). MS (ESI): $\mathrm{m} / z$ (\%) 243 (16) [M+1], 197 (42), 168 (80), 167 (100). Anal. Calcd for $\mathrm{C}_{14} \mathrm{H}_{13} \mathrm{NO}$ : C, 79.59; H, 6.20; N, 6.63. Found: C, 79.42; H, 6.36; N, 6.55.

4-(3-Nitrophenylamino)benzaldehyde (3t). Yellow solid; mp 152-155 ${ }^{\circ} \mathrm{C} .{ }^{1} \mathrm{H} \mathrm{NMR}(300 \mathrm{MHz}$, $\left.\mathrm{CDCl}_{3}\right): \delta 6.35$ (s, 1H; NH), 7.16, $7.13\left(\mathrm{AA}^{\prime} ; 2 \mathrm{H} ; \mathrm{H}_{\mathrm{Ar}}\right), 7.49-7.52\left(\mathrm{~m}, 2 \mathrm{H} ; \mathrm{H}_{\mathrm{Ar}}\right), 7.85,7.82\left(\mathrm{XX}^{\prime}\right.$; $\left.2 \mathrm{H} ; \mathrm{H}_{\mathrm{Ar}}\right), 7.87-8.04\left(\mathrm{~m}, 2 \mathrm{H} ; \mathrm{H}_{\mathrm{Ar}}\right), 9.87(\mathrm{~s}, 1 \mathrm{H} ; \mathrm{CHO}) .{ }^{13} \mathrm{C} \mathrm{NMR}\left(75 \mathrm{MHz}, \mathrm{CDCl}_{3}\right): \delta 114.1$ $(\mathrm{CH}), 116.1(\mathrm{CH}), 117.6(\mathrm{CH}), 122.3(\mathrm{C}), 125.2(\mathrm{C}), 130.4(\mathrm{C}), 132.1(\mathrm{CH}), 142.1(\mathrm{C}), 147.6$ (C), 148.5 (C), 190.3 (CHO).

\section{Acknowledgements}

We would like to thank The Project Sponsored by the Scientific Research Foundation for the Returned Overseas Chinese Scholars, State Education Ministry of China, and National Natural Science Foundation of China (21002081) for financial support.

\section{References}

1. Lawrence, S. A. Amines: Synthesis Properties and Application, Cambridge University: Cambridge, 2004.

2. Suwanprasop, S.; Nhujak, T.; Roengsumran, S.; Petsom, A. Ind. Eng. Chem. Res. 2004, 43, 4973.

3. Ackermann, L.; Althammer, A. Angew. Chem. Int. Edit. 2007, 46, 1627.

4. Anderson, K. W.; Tundel, R. E.; Ikawa, T.; Altman, R. A.; Buchwald, S. L. Angew. Chem. Int. Edit. 2006, 45, 6523.

5. Bedford, R. B.; Betham, M.; Charmant, J. P. H.; Weeks, A. L. Tetrahedron 2008, 64, 6038.

6. Bedford, R. B.; Cazin, C. S. J. Chem. Comm. 2002, 2310.

7. Fors, B. P.; Watson, D. A.; Biscoe, M. R.; Buchwald, S. L. J. Am. Chem. Soc. 2008, 130, 13552.

8. Nandakumar, M. V.; Verkade, J. G. Angew. Chem. Int. Edit. 2005, 44, 3115.

9. Sapountzis, I.; Knochel, P. J. Am. Chem. Soc. 2002, 124, 9390.

10. Wolfe, J. P.; Tomori, H.; Sadighi, J. P.; Yin, J. J.; Buchwald, S. L. J. Org. Chem. 2000, 65, 1158.

11. Biscoe, M. R.; Fors, B. P.; Buchwald, S. L. J. Am. Chem. Soc. 2008, 130, 6686.

12. Surry, D. S.; Buchwald, S. L. J. Am. Chem. Soc. 2007, 129, 10354.

13. Wolfe, J. P.; Buchwald, S. L. J. Org. Chem. 1997, 62, 6066.

14. Greiner, A. Tetrahedron Lett. 1989, 30, 931.

15. Wadia, M. S.; Patil, D. V. Synth. Commun. 2003, 33, 2725. 
16. Acemoglu, M.; Allmendinger, T.; Calienni, J.; Cercus, J.; Loiseleur, O.; Sedelmeier, G. H.; $\mathrm{Xu}, \mathrm{D}$. Tetrahedron 2004, 60, 11571.

17. Selvakumar, N.; Srinivas, D.; Azhagan, A. M. Synthesis 2002, 2412.

18. Kam, L. E.; Grimaud, L.; Oble, J. Angew. Chem. Int. Ed. 2005, 44, 7961.

19. Yang, H.; Li, Z.-B.; Shin, D.-S.; Wang, L.-Y.; Zhou, J.-Z.; Qiao, H.-B.; Tian, X.; Ma, X.-Y.; Zuo, H. Synlett. 2010, 483.

20. Desmarets, C.; Schneider, R.; Fort, Y. J Org. Chem. 2002, 67, 3029.

21. Ma, C.; Zhang, Q.; Ding, K.; Xin, L.; Zhang, D. J. Tetrahedron Lett. 2007, 48, 7476.

22. Guram, A. S.; Buchwald, S. L. J. Am. Chem. Soc. 1994, 116, 7901.

23. Reddy, C. V.; Kingston, J. V.; Verkade, J. G. J. Org. Chem. 2008, 73, 3047.

24. Altman, R. A.; Anderson, K. W.; Buchwald, S. L. J. Org. Chem. 2008, 73, 5167.

25. Saavedra, C.; Hernández, R.; Boto, A.; A’ lvarez, E. J. Org. Chem. 2009, 74, 4720.

26. Gao, C. Y.; Yang, L. M. J. Org. Chem. 2008, 73, 1624.

27. Shen, Q. L.; Hartwig, J. F. Org. Lett. 2008, 10, 4109.

28. Kabalka, G. W.; Zhou, L. L. Lett. Org. Chem. 2006, 3, 320.

29. Hughes, G. M. K.; Saunders, B. C. J. Chem. Soc. 1956, 20, 3814.

30. Artamkina, G. A.; Sergeev, A. G.; Shtern, M. M.; Beletskaya, I. P. J. Org. Chem. 2006, 42, 1683.

31. McNulty, J.; Cheekoori, S.; Bender, T. P.; Coggan, J. A. Eur. J. Org. Chem. 2007, 9, 1423.

32. Wadia, M. S.; Patil, D. V. Synth. Commun. 2003, 33, 2725.

33. Fukuzaki, E.; Nishide, H. J. Am. Chem. Soc. 2006, 128, 996.

34. Brown, U.M.; Carter, P. H.; Tomlinson, M. J. Chem. Soc. 1958, 9, 1843. 